\title{
Um Modelo de Pilha Elétrica
}

\author{
Modeling a battery \\ G. F. Leal Ferreira \\ Instituto de Física de São Carlos, USP, CP 369, 13560-970, São Carlos, SP \\ guilherm@if.sc.usp.br
}

Recebido em 06 de fevereiro, 2003. Aceito em 28 de maio, 2003.

\begin{abstract}
O funcionamento de uma pilha elétrica é modelado através de um poço duplo de potencial entre os quais age força de natureza físico-química em portadores ativos, preferenciando um dos sítios. Com isso, desenvolve-se uma diferença de potencial entre os mesmos, fonte da força eletromotriz. A circulação externa de carga causa diminuição da força eletromotriz através de mecanismo adequado. Calcula-se o seu valor em função da carga circulada e a cinética de recuperação em circuito aberto após ser submetida a breve curto-circuito.
\end{abstract}

The functioning of a battery is modeled through a potential double well in which a force of physico-chemical origin acts on charges, causing the population on one side side to increase in relation to the other. The electrical potential thus created is the source of an electro motive force. External charge circulation makes this electro motive force to decrease by means of an adequate mechanism. Its value as a function of the amount of circulated charge is calculated, as well as the kinetics of recovering after briefly short-circuiting it.

\section{Introdução}

Apresentamos neste artigo modelo que procura reproduzir, de forma simplificada, o funcionamento de uma pilha. Invocaremos o modelo do duplo poço de potencial, originalmente introduzido por Fröhlich [1] no estudo de dielétricos sólidos. No caso presente, o duplo poço de potencial representará a interface entre espécies químicas no interior da pilha. A população de uma espécie elétrica ativa flutua termicamente entre os poços. A população, porém, não se reparte igualmente entre eles porque uma força natural - de origem físico-química -, age preferencialmente num sentido, fazendo com que se estabeleça uma diferença de potencial entre os poços - ou polos da pilha -, que dá origem à sua força eletromotriz. Se os polos forem ligados externamente, fluirá carga entre eles, o que interferirá na cinética intra-poços. Porém, para que o modelo não viole a $2^{a}$ Lei da Termodinâmica - predizendo a realização contínua de trabalho a temperatura constante -, devemos impor que a circulação externa de carga implica em diminuição correspondente de população ativa nos poços, através de mecanismo adequado. Da circulação externa também resulta então a diminuição da força eletromotriz da pilha, o que explica a máxima na prática com pilhas-padrão, de que a passagem de corrente deve ser evitada para não prejudicar a sua performance como padrão. Na seção II, apresentaremos o modelo do duplo poço de potencial, como usado na teoria da polarização elétrica e na seção III o adaptaremos ao estudo da pilha, deduzindo as equações cinéticas do modelo. Na IV, o estado de equilíbrio, com a máxima força eletromotriz resultante é obtida. Na seção V, mostra-se que a minimização da energia livre, através de raciocínio termodinâmico-mecânico-estatístico, produz o mesmo resultado que o da seção anterior. Na seção VI o comportamento qualitativo de pilhas é brevemente relembrado e as previsões quantitativas do modelo são calculadas. Comentários encerram o artigo na seção VII.

\section{O modelo do duplo poço de poten- cial}

No modelo de Fröhlich [1], ver também [2], o dipolo elétrico em matriz sólida é representado por uma, vamos dizer, carga negativa, ladeada por dois sítios capazes de alojar uma carga positiva. Esta flutua entre as duas posições, separadas por uma barreira de potencial, que deve ser vencida por ativação térmica. O comportamento médio de uma população de dipolos como estes, é então analisado. No caso dielétrico, estuda-se a perturbação na ocupação dos sítios, ou seja a polarização, causada pela aplicação de um campo elétrico, ver Fig. 1. Em conformidade com o sentido do campo elétrico mostrado, do poço $I$ ao poço $I I$, a população do poço $I I$ tende a crescer em detrimento da do poço $I$, já que o número total de dipolos é tomado como constante. As frequências de passagem de cargas do poço $I$ ao poço $I I$, $\nu_{1,2}$, e do poço $I I$ ao poço $I, \nu_{2,1}$, são

$$
\begin{aligned}
& \nu_{1,2}=\nu_{0} e^{-\left(\phi-\frac{e E a}{2}\right) / k T} \\
& \nu_{2,1}=\nu_{0} e^{-\left(\phi+\frac{e E a}{2}\right) / k T}
\end{aligned}
$$


em que, ver Fig. 1, $\nu_{0}$ é a chamada freqüência de escape, $\phi$ é a barreira de potencial, $E$ o campo elétrico aplicado, $2 a$ a distância entre o fundo dos poços, $e$ a carga elementar, $k$ a constante de Boltzmann e $T$ a temperatura absoluta. $\mathrm{O}$ fator $e E a / 2$ dá o efeito do campo elétrico, abaixando a barreira de 1 para 2 e aumentando de 2 para 1 . As equações de balanço entre as populações dos poços, $N_{1}$ e $N_{2}$, são

$$
\begin{aligned}
& \frac{d N_{1}}{d t}=-\nu_{1,2} N_{1}+\nu_{2,1} N_{2} \\
& \frac{d N_{2}}{d t}=-\nu_{2,1} N_{2}+\nu_{1,2} N_{1}
\end{aligned}
$$

Com $N_{1}+N_{2}=N_{0}$, número total de dipolos, uma das equações, Eq. 3 ou Eq. 4, pode, em princípio, ser integrada. No caso dielétrico, eEa/2 é, em geral, pequeno em comparação com $k T$, e os expoentes nas Eqs. 1 e 2 podem ser linearizados na parte dependente de $e a E / 2 k T$. Além disso, definindo a polarização elétrica total como $P=e a\left(N_{2}-N_{1}\right)$, a Eq. 3 dá origem à famosa equação de Debye [2]

$$
\frac{d P}{d t}=2 \nu\left(-P+N_{0} e^{2} a^{2} E / k T\right)
$$

com

$$
\nu=\nu_{0} e^{-\frac{\phi}{k T}}
$$

Na próxima seção adaptaremos o modelo do duplo poço de potencial para a descrição do funcionamento da pilha elétrica.

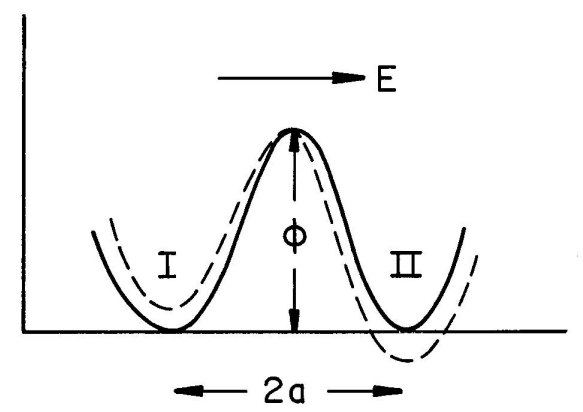

Figura 1. Modelo de Fröhlich para a polarização dielétrica. O campo aplicado $E$ abaixa a barreira de $I$ para $I I$ aumenta no sentido oposto. $\mathrm{O}$ traço cheio representa o potencial antes da aplicação do campo, o tracejado, depois.

\section{O modelo do duplo poço e a pilha}

Usaremos o modelo do duplo poço de potencial com a seguinte interpretação e acessórios. Ao longo da área de separação (interface) entre dois meios (esquema de perfil na Fig. 2) há $N_{0}$ sítios com duplos poços, alojando cada duplo poço um portador ativo (que 'vê' o duplo poço), tomado como positivo. Há também, de cada lado da interface, $N_{0} / 2$ cargas negativas de compensação. Assim, os poços $I$ e $I I$ contêm $N_{1}$ e $N_{2}$ portadores positivos ativos, respectivamente e, em média, $N_{0} / 2$, cada, portadores negativos, que com liberdade de linguagem, admitiremos pertencer aos poços $I$ e $I I$. O excesso de carga em $I$ será, por exemplo, $N_{1}-N_{0} / 2$. Campo de natureza físico-química, $F$, age de $I I$ para $I$ e assim $I$ acumula portadores ativos positivos e seu potencial elétrico cresce em relação ao do sítio $I I$. Com isso, aparece campo elétrico $E$ entre os dois lados da interface, sendo $F-E$ o campo total, contado no sentido de $I I$ para $I$. Para estudarmos a situação em que a pilha fornece corrente, introduzimos também as cargas $Q_{2}$, que se acumulam no exterior de $I I$ e que são originárias do processo de condução externa e, portanto, devido diretamente à diminuição da população de $N_{1}$. As cargas $Q_{2}$, embora criem campo elétrico como as $N_{2}$,não participam do processo intra-poços. A consideração dessa carga $Q_{2}$ que se torna inativa ao circular externamente, é importante porque, de outra forma, a pilha viria a violar a $2^{a}$ Lei da Termodinâmica através do procedimento seguinte. A partir da situação de equilíbrio a uma dada temperatura, deixaríamos a pilha funcionar durante certo tempo, realizando algum trabalho. Neste processo, a população $N_{1}$ diminuiria e $N_{2}$ aumentaria. Interromperíamos o fluxo e esperaríamos tempo suficiente para que o equilíbrio fosse restaurado no duplo poço, voltando à situação inicial de equilíbrio. Repetindo o processo, estaríamos obtendo trabalho às custas de energia térmica retirada de fonte a uma única temperatura, o que a $2^{a}$ Lei não permite. O processo de acumulação de cargas $Q_{2}$ garante que a pilha tenha capacidade finita de circular carga - maior ou menor conforme a área da interface -, o que está de acordo com a nossa intuição. A pilha será reversível se for possível, através de trabalho elétrico, fazer $Q_{2}$ retornar ao poço $I$.

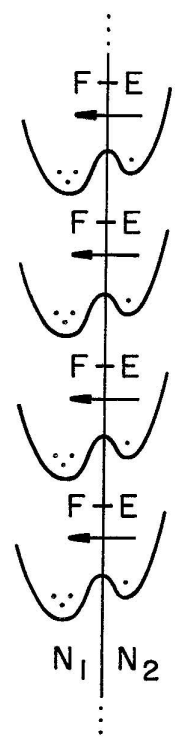

Figura 2. Os sítios ao longo da interface entre os meios ativos, são representados por duplos poços de potencial, assentados nos dois meios. Como o campo total, $F-E$, age para a esquerda, a população, simbolizada por pontos, no interior do poço $I I, N_{2}$, é maior que a do poço $I, N_{1}$. As cargas de compensação não estão mostradas, como também as cargas circuladas, que se tornaram inativas, $Q_{2}$. 
Vamos agora escrever a equação de balanço, semelhante às Eqs. 3 e 4 acima, levando em conta a possibilidade de troca de carga através de corrente externa. Antes, porém, re-escrevemos as frequências de passagem de portadores de $I$ para $I I$, e vice-versa

$$
\begin{aligned}
& \nu_{1,2}=\nu e^{(-F+E) e a / 2 k T} \\
& \nu_{2,1}=\nu e^{(F-E) e a / 2 k T},
\end{aligned}
$$

sendo $\nu$ dado pela Eq. 6 .

\section{III.1 As equações de balanço e o campo elétrico}

As equações de balanço, supondo que uma corrente externa $i$ circula de $I$ para $I I$, são:

$$
e \frac{d N_{1}}{d t}=-i-\nu_{1,2} N_{1}+\nu_{2,1} N_{2}
$$

e

$$
\begin{gathered}
e \frac{d N_{2}}{d t}=-\nu_{2,1} N_{2}+\nu_{1,2} N_{1} \\
\frac{d Q_{2}}{d t}=i
\end{gathered}
$$

Devemos calcular o campo elétrico $E$, que comparece nos expoentes das Eqs. 7 e 8 , e que depende de $N_{1}$ e de $N_{2}$. Notemos que as cargas totais nos poços $I$ e $I I, q_{1}$ e $q_{2}$, incluindo as inativas negativas, são, respectivamente,

$$
q_{1}=e\left(N_{1}-\frac{N_{0}}{2}\right) \text { e } q_{2}=e\left(N_{2}-\frac{N_{0}}{2}\right)+Q_{2}
$$

Como temos neutralidade no total, $q_{1}+q_{2}=0$, segue que o campo elétrico $E$ é o campo entre duas distribuições de carga, de densidades $+q_{1} / A \varepsilon_{0}$ e $-q_{1} / A \varepsilon_{0}$,

$$
E=\frac{q_{1}}{A \varepsilon_{0}}=\frac{e\left(N_{1}-\frac{N_{0}}{2}\right)}{A \varepsilon_{0}},
$$

$\operatorname{com} \varepsilon_{0}$ a permitividade do vácuo e $A$ a área da interface.

\section{A pilha em circuito aberto}

Supondo circuito aberto (isto é, $i=0$ ) e as populações dos poços em equilíbrio, vamos calcular a diferença de potencial entre os polos (ou poços). Como

$$
e N_{1}+e N_{2}+Q_{2}=e N_{0}
$$

e definindo para o problema presente $P$ como

$$
P=e\left(N_{1}-N_{2}\right)
$$

podemos escrever para $N_{1}$ e $N_{2}$

$$
\begin{gathered}
N_{1}=\frac{e N_{0}-Q_{2}+P}{2 e} \\
N_{2}=\frac{e N_{0}-Q_{2}-P}{2 e} .
\end{gathered}
$$

Notemos que o valor máximo de $P$, o qual ocorre para $Q_{2}=0$, é a carga que a pilha pode fornecer externamente. Em circuito aberto, $i=0$ e $Q_{2}$ tem valor constante. As Eqs. 9 e 10, tendo em vista as Eqs. 16 e 17, fornecem

$$
P\left(\nu_{12}+\nu_{21}\right)=\left(e N_{0}-Q_{2}\right)\left(\nu_{21}-\nu_{12}\right),
$$

ou, em vista das Eqs. 9-11

$$
P=\left(e N_{0}-Q_{2}\right) \operatorname{tgh}\left[(F-E) \frac{e a}{2 k T}\right]
$$

Pelas Eqs. 13 e 16, o campo elétrico é

$$
E=\frac{P-Q_{2}}{2 \varepsilon_{0} A}
$$

\section{IV.1 A força eletromotriz da pilha}

A força eletromotriz, f.e.m., $\epsilon\left(Q_{2}\right)$, é a diferença de potencial entre os polos (poços) em circuito aberto, depois que uma carga $Q_{2}$ circulou externamente. Ela, em termos de $P$, Eq.14, é

$$
\epsilon\left(Q_{2}\right)=E a=\frac{\left(P-Q_{2}\right) a}{2 \varepsilon_{0} A} .
$$

Chamamos de f.e.m. da pilha, $\epsilon$, o valor máximo de $\epsilon\left(Q_{2}\right)$, ou seja, $\epsilon(0)$, que pode ser obtido da Eq. 19, de forma implícita,

$$
\epsilon=\frac{Q}{2 C} \operatorname{tgh}\left[(a F-\epsilon) \frac{e}{2 k T}\right],
$$

com

$$
Q=e N_{0} \quad \text { e } C=\frac{\varepsilon_{0} A}{a},
$$

sendo $Q$ a carga e $C$ a capacidade geométrica da interface. Em termos de $P$ e chamando de $P_{e q}$ o correspondente valor de equilíbrio, temos, das Eqs. 19 e 20,

$$
P_{e q}=e N_{0} \operatorname{tgh}\left[\left(F-\frac{P_{e q}}{2 \varepsilon_{0} A}\right) \frac{e a}{2 k T}\right] .
$$

Na seção seguinte, procuraremos obter $P_{e q}$ através de raciocínio termodinâmico.

\section{Minimizando a energia livre}

Obtivemos na Eq. 24 a 'polarização' entre os polos da pilha para $Q_{2}=0$, partindo das Eqs. 7-9, isto é, por raciocínio cinético. Como exercício, procuraremos agora obter a mesma 'polarização' por raciocínio termodinâmico. Em geral, a energia livre $H$ é a diferença entre a energia $U$ e $T$ vezes a entropia $S$ do sistema [3]. Expressando $U$ e $S$ em termos de uma das variáveis, vamos dizer $P$, e minimizando $H$ em relação a $P$, devemos encontrar o estado de equilíbrio, obtido de forma independente da anterior.

A energia $U$ pode ser considerada composta de dois termos. Uma, $U_{1}$, é a energia físico-química dos portadores ativos e a outra, $U_{2}$, é a energia elétrica criada pela separação 
de cargas. Quanto à primeira, $U_{1}$, tomando como ponto de referência o centro da barreira, é

$$
U_{1}=e N_{2} F \frac{a}{2}-e N_{1} F \frac{a}{2}=-\frac{P F a}{2}
$$

raciocinando para o campo $F$ como fazemos para o campo $E$, no cálculo da energia potencial. A energia eletrostática $U_{2}$ será a energia do condensador plano carregado, de área $A$, espessura $a$ e densidade de energia $\varepsilon_{0} E^{2} / 2$, que, em termos de $P$, é

$$
U_{2}=\frac{a P^{2}}{8 \varepsilon_{0} A} .
$$

A entropia $S$ é $k \ln \left(N_{0}\right.$ ! / $\left(N_{1} ! N_{2}\right.$ !) que na aproximação de Stirling e expressa em termos de $N_{0}$ e $P$ é

$$
\begin{aligned}
S=k[ & N_{0} \ln N_{0}-\left(N_{0}+P / 2 e\right) \ln \left(N_{0}+P / 2 e\right) \\
& \left.-\left(N_{0}-P / 2 e\right) \ln \left(N_{0}-P / 2 e\right)\right] .
\end{aligned}
$$

Minimizando $H=U_{1}+U_{2}-T S$, a $T$ constante, em relação a $P$, encontra-se, $\operatorname{com} P=P_{e q}$,

$$
-\frac{a F}{2}+\frac{a P_{e q}}{4 \varepsilon_{0} A}-k T \ln \frac{e N_{0}-P_{e q}}{e N_{0}+P_{e q}}=0
$$

de onde resulta a Eq. 24 .

\section{Sobre a performance de uma pi- lha}

\section{VI.I. Qualitativo}

Apesar do emprego praticamente universalizado de pilhas elétricas, a sua performance durante uso não é comentada na literatura. Pessoalmente, o autor lembra-se que numa experiência informal monitorada pelos Srs. Salvador B. Sanchez Vera, Carlos Alberto Trombella e Sebastião B. Pereira, na qual se mediu a corrente no circuito de lanterna (duas pilhas comuns de $1,5 \mathrm{~V}$ em série, lâmpada e amperímetro) durante dois dias, a corrente caiu lenta mas constantemente ao longo do tempo. Um outro fato conhecido é que se produzimos um breve curto-circuito entre os polos, a f.e.m. da pilha cai mas recupera-se em circuito aberto (provavelmente a um valor inferior ao inicial). Na sub-seção seguinte, procuraremos obter expressões para a f.e.m. em função da carga $Q_{2}$ circulada externamente e a mencionada recuperação da f.e.m. após breve curto.

\section{VI.II. Quantitativo}

Vamos quantificar o modelo e ao fazê-lo teremos de fazer escolhas. Para começar, olhemos as Eqs. 22 e 23. Por elas, a f.e.m. $\epsilon$ da pilha depende dos parâmetros $Q / 2 C$, $a F$ e $e / k T$, que podem ser reduzidos a dois tomando-se $\epsilon_{m}=Q / 2 C$ como unidade de potencial. Mas $\epsilon_{m}=Q / 2 C$, pela Eq. 23, é $e a N_{0} / 2 \varepsilon_{0} A$ e $N_{0} / A$ deve ser uma fração da densidade de sítios superficiais na interface ou seja da ordem de $10^{20} / \mathrm{m}^{2}$, vamos dizer, $n \cdot 10^{20} / \mathrm{m}^{2}$. Como $a \approx 1 \AA$
$=10^{-10} \mathrm{~m}$, temos $\epsilon_{m} \approx 10^{2} n$. Se tomarmos $\epsilon_{m}$ como da ordem de $1 \mathrm{~V}$, obteremos para $n$ o valor aceitável de $1 \%$. Prosseguindo na busca de valores razoáveis para os parâmetros, vamos escrever a Eq. 22 tendo $\epsilon_{m}$ como unidade de potencial,

$$
\frac{\epsilon}{\epsilon_{m}}=\operatorname{tgh}\left[\left(\frac{a F}{\epsilon_{m}}-\frac{\epsilon}{\epsilon_{m}}\right) K\right]
$$

com

$$
K=\frac{e \epsilon_{m}}{2 k T} .
$$

Para $\epsilon_{m} \approx 1 \mathrm{~V}, K$ na Eq. 30 será da ordem de 20. Vê-se que com este alto valor de $K, \epsilon$ será $\simeq \epsilon_{m}$ para $a F \gtrsim \epsilon_{m}$. Neste caso, $\epsilon$ não dependerá da temperatura e também não dependerá do próprio valor de $F$, devido à limitação do valor da tangente hiperbólica. Tomaremos este limite como desinteressante ou não físico e escolheremos a outra alternativa que é supor que $a F<\epsilon_{m}$. Seja então $f=a F / \epsilon_{m}$, com $f<1$. Com $K$ grande, e chamando $\epsilon / \epsilon_{m}$ de $y$, isto é, $y=\epsilon / \epsilon_{m}$, a Eq. 29 se escreve como

$$
y=\operatorname{tgh}[K(f-y)\}]
$$

que, $\operatorname{com} w=f-y$, fica

$$
f-w=\operatorname{tgh}(K w) .
$$

Com $K$ grande, podemos aproximar a tgh como $K w$, para $w$ entre 0 e $1 / K$ e por 1 para $w>1 / K$. Como estamos no primeiro caso, obtemos

$$
f-w \simeq K w
$$

$\mathrm{ou}$

$$
\epsilon \simeq a F\left(1-\frac{1}{K}\right) .
$$

Como $1 / K$ é diretamente proporcional a $T$, vê-se que $\epsilon$ decresce com o aumento de $T$.

Para estudarmos o que ocorre para $Q_{2}>0$, devemos retornar à Eq. 21, usando a Eq. 19:

$$
\epsilon\left(Q_{2}\right)=\epsilon_{m}\left\{\left[1-Q_{2}^{\prime}\right] \operatorname{tgh}\left[\left(a F-\epsilon\left(Q_{2}\right) \frac{e}{2 k T}\right]-Q_{2}^{\prime}\right\}\right.
$$

com

$$
Q_{2}^{\prime}=\frac{Q_{2}}{e N_{0}}
$$

No mesmo espírito da aproximação anterior levando à Eq. 35, obtem-se para $\epsilon\left(Q_{2}\right)$

$$
\epsilon\left(Q_{2}\right)=a F-\frac{1}{K\left(1-Q_{2}^{\prime}\right)}\left(a F+\epsilon_{m} Q_{2}^{\prime}\right)
$$

que patentemente decresce para $Q_{2}^{\prime}$ crescente.

Para o estudo da recuperação da voltagem após breve curto, mencionado no fim da subseção anterior, temos que voltar às Eqs. 9 e 10 e usar as Eqs. 15-17. Elegendo $\epsilon\left(Q_{2}, t\right)$ como variável independente e com $i=0$, circuito aberto, obtemos para a cinética em busca do equilíbrio, após circulação total de $Q_{2}$,

$$
\frac{d \epsilon\left(Q_{2}, t\right)}{d t}=2 \epsilon_{m} v \operatorname{senh}\left[\left(F a-\epsilon\left(Q_{2}, t\right) \frac{e a}{2 k T}\right]\right.
$$




$$
-2 \epsilon\left(Q_{2}, t\right) v \cosh \left[(F-E) \frac{e a}{2 k T}\right]-2 \epsilon_{m} Q_{2}^{\prime} .
$$

$\mathrm{Na}$ aproximação em que estamos trabalhando, de linearização da tangente hiperpólica, o seno hiperbólico também pode ser linearizado enquanto que o cosseno pode ser substituido por 1. A Eq. 38 dá então, após manipulação,

$$
\begin{gathered}
\frac{d \epsilon\left(Q_{2}, t\right)}{2 v d t}+\left[\left(1-Q_{2}^{\prime}\right) K+1\right] \epsilon\left(Q_{2}, t\right)= \\
\left(1-Q_{2}^{\prime}\right) K a F-\epsilon_{m} Q_{2}^{\prime} .
\end{gathered}
$$

que mostra que o valor de equilíbrio, $\epsilon\left(Q_{2}\right)$, Eq. 39, é alcançado exponencialmente, com a constante de tempo essencialmente igual a $\left[2 v\left(1-Q_{2}^{\prime}\right) K\right]^{-1}$, isto é, tão mais lentamente quanto mais descarregada a pilha estiver.

\section{Comentários finais}

As previsões quantitativas do modelo, calculadas na seção anterior, são razoáveis. O modelo, de forma muito simplificada, reproduz o número mínimo de três elementos que uma pilha deve conter: dois eletródios diferentes mergulhados num meio ativo (a ligação externa para passagem de corrente poderia ser feita com prolongamento de um dos eletródios até o outro contacto). A força de natureza físicoquímica simbolizaria a ação do meio ativo agindo entre os dois eletródios, representado pelos dois poços. Falta ao modelo a resistência interna da pilha, que na prática pode jogar papel importante no estágio de envelhecimento, mas não é fundamental.

\section{Agradecimentos}

O autor agradece ao árbitro pela correção de inúmeras fórmulas e outras sugestões.

\section{Referências}

1. H. Fröhlich, Theory of Dielectrics, Oxford at Clarendon Press, 1949, Cap. II.

2. A. J. Dekker, Solid State Physics, Prentice-Hall, 1962, Cap. 6.

3. G. W. Castellan, Físico-Química, trad. Luiz Carlos Guimarães, Livros Técnicos e Científicos, 1977, Cap. 10. 